\title{
Dental Practitioners' Opinions and Methods in the Management of Oral Mucosal Lesions and TMJ Disorders in Dakshina Kannada District: A Questionnaire Based Survey
}

INTRODUCTION: Oral mucosal lesions and temperomandibular joint (TMJ) disorders are important areas of concern in the present dental practice due to their increasing prevalence. Timely management is important as it aids in maximal prognosis.

AIMS AND OBJECTIVES: The aim of the study was to determine the awareness of dental practitioners in Dakshina Kannada district of Karnataka, regarding various aspects of oral mucosal lesions and TMJ disorders. And also to know their interest in managing such disorders.

MATERIALS AND METHODS: A self- administered questionnaire was constructed and given to dental practitioners, selected through multistage cluster random sampling method; from Dakshina Kannada district. Chi-square test was performed and the level of significance was set at $\mathrm{p}<0.05$.

RESULTS: The majority of dental practitioners had a dental practice of duration five to ten years. Many of the respondents (72\%) indicated that oral ulcers are the most common mucosal lesions and prescribed topical anaesthetics for these. The investigations suggested were blood investigations (24\%) for various oral mucosal lesions and radiographs $(20 \%)$ for TMJ disorders. Different pharmacological treatments provided by the majority of the respondents were topical anaesthetics and multi vitamin supplements. About $34 \%$ dental practitioners routinely refer the patients to specialty practitioners.

CONCLUSION: The present study shows that there should be emphasis on workshops and various other awareness programs for general dental practitioners for better understanding of the subject and about the latest protocols regarding referral of the patients to specialists.

KEYWORDS: Dental Practitioners, Oral Mucosal Lesions, TMJ Disorders

\section{INTRODUCTION}

Mucosal diseases involving the oral cavity encompass various common conditions. Majority of these present with comparable features that can make diagnosis difficult to accomplish based only on clinical examination. These lesions may be assorted based on different clinical parameters, such as acute or chronic conditions, single or multiple lesions, primary or recurrent nature, and/or local or generalized disease. ${ }^{1}$

Temporomandibular disorders (TMDs) are a collective term embracing a number of clinical problems that involve the masticatory muscles, the temporomandibular joints and associated structures, or both. ${ }^{2}$ The etiological factors for TMDs are numerous and mainly related to emotional pressure, occlusal abnormalities, teeth loss, postural variation, masticatory muscular dysfunction, internal and external changes in TMJ structure and the various combinations of factors. ${ }^{3}$

Epidemiological studies have shown that mucosal lesions of the oral cavity and temporomandibular joint(TMJ) disorders are accelerating worldwide. ${ }^{4,5}$ The exact reasons for these epidemiological changes are obscure but may be the after effect of changing life style and due to psychosocial instabilities. Efficacious management of the disorders can be assured by an early diagnosis that leads to maximal prognosis. The current scenario also necessitates a proper communication and referral of these patients to speciality dental practitioners although it is known that there can be significant referral delay. ${ }^{6}$

The aim of the study was to assess the knowledge and awareness among dental practitioners of Dakshina Kannada regarding relevant aspects of oral mucosal lesions and TMJ disorders.

\section{MATERIALS AND METHODS}

The present descriptive study was designed and conducted to assess the knowledge and awareness of Dental practitioners towards oral mucosal lesions and TMJ disorders. Ethical clearance for the study was obtained by the institutional Ethics Committee (IEC KVGDCH). All willing participants who satisfied the inclusion/ exclusion criteria were personally approached with a prior appointment and necessary data was collected from them. Informed consent was obtained by the participants before the commencement of the study. The study group included all willing Dental Practitioners having a registered Dental practice of at least one year within Dakshina Kannada District. In clinics with more than one registered dental practitioner, the most senior practitioner was included in the study. Practitioners 
with a master's degree in the subject of Oral Medicine and Radiology and specialist consultants visiting clinics for speciality treatment were excluded from the study. Data was collected using a selfadministered questionnaire comprising two parts Part A recorded the basic demography of the participants and Part B was a pre tested and validated questionnaire containing a list of 8 factual and higher order questions including the most common, occasional and rare oral mucosal lesions and TMJ disorders they witnessed per month, their frequencies, various investigations advised, treatment provided by them and regarding referral pattern and frequencies of these patients to specialty dental practitioners. Initially a list of 20 questions was developed through literature review and a content validation exercise was performed by mailing the questions to five specialists and their opinions on the validity of the questions were sought on a five point Likert scale, the responses were summed and a content validation ratio was calculated, questions with a content validation ratio of 0.6 and more were included in the final questionnaire. (Figure 1). The sample size was estimated based on the pilot study for the management of oral mucosal lesions and TMJ disorders among the dental practitioners, at an estimated prevalence of $59 \%$ for a finite sample of 638 registered dental practitioners at precision rate of $1 \%$ and a confidence level of $2 \%$, the estimated sample size was 182 , a five percent of the estimated sample size was added to compensate for sampling loss if any and the sample size was rounded off to 200 . A list of registered dental practitioners was obtained from the offices of the of Karnataka private medical establishment, DK Branch. The list was segregated into five administrative divisions of DK district (Sullia, Puttur, Belthangady, Mangaluru and Bantwal), from among a total of 638 registered dental practitioners a sample proportionate to size was drawn from each of the administrative divisions. The sampling frame of the included dental practitioners is as shown in Figure 2. The practitioners were approached in person. The purpose of the study was explained and the necessary data was collected the same /consecutive day.

Analysis of data was performed using SPSS software version 18 , descriptive statistics were calculated and expressed as percentages. A chi-square analysis was preformed to find out the significance in the results in relation to demographic characteristics included in the study. P value was set at less than 0.05 .

\section{RESULTS}

Fifty two percent of dental practitioners had a dental practice of more than ten years. Seventy two percent of the respondents reported that oral ulcers are the most common oral mucosal lesions, they encountered in their practice. Oral cancer was reported as the least common lesion by $26 \%$ of the respondents (Table 1).

About $56 \%$ of the respondents reported that they have witnessed up to five white and red lesions per month. But a very small group could suggest the presence of tumors in their patient population (Table 2).Most of the practitioner utilized visual examination to arrive at a diagnosis. Only one percent of them advised for light based examination methods for diagnosis. Twenty four percent of the practitioners advised for blood investigations to rule out underlying systemic conditions (Figure 2).

Majority (97\%) of them provided various pharmacological modalities for the treatment of various oral mucosal diseases. Topical applications of analgesics and anaesthetics were advised for most oral mucosal lesions (Table 3 ). Thirty four percent of the respondents reported that they routinely refer their patients to specialty practitioners in case of suspicious diseases (Figure 3).

Forty one percent of the practitioners refer their patients with different white and red lesions to an oral physician while others prefer an oral surgeon (Figure 4).

TMJ disorders were reported as rare by $24 \%$ of the respondents (Table 1). About five percent of the respondents reported that they have witnessed less than five such conditions per month (Table 2). Appliance therapy was given by three percent of the practitioners for various TMJ disorders (Table 4). About four percent of the respondents reported that they referred patients with TMJ disorders to orthopaedic and ENT surgeon (Figure 4).

\section{DISCUSSION}

The present study indicates the interest and methods in managing oral mucosal lesions and TMJ disorders by general dental practitioners.

The practitioners mostly witness patients with various mucosal lesions and TMJ abnormalities in their clinical practice. Screening, in medicine, is a 
strategy used in population to identify disease in individuals without the presence of signs or symptoms of that disease or examination of a group of asymptomatic individuals to identify those with a high probability of having or developing a given disease. Recognition of an abnormality is the pivotal step in early detection of lesions. It is vital as it helps to provide interventional measures, halting the progress of oral mucosal diseases. ${ }^{7}$

Chronic pain conditions involving the orofacial region are difficult to diagnose due to the interrelationship of structures in the area. TMJ abnormalities may be overlooked due to lack of knowledge. ${ }^{8}$

Visual examination used for the diagnosis by most of the dental practitioners only helps in initial screening, but not in confirming the diagnosis. ${ }^{9}$ Moreover, the reliability of visual examination is variable as it depends on the training and skill of the clinician. Only 2 percent advised an exfoliative cytology and only 17 percent of the respondents advised a biopsy for premalignant oral lesion. Tissue biopsy is an invasive, expensive, and often timeconsuming investigation. There also exists both inter and intra-observer variability in the diagnostic interpretation of the tissue sample. ${ }^{7}$ This may be the reason for the low rate in the advice and performance of biopsy in the study. Thus, there is evolving need for simple and reliable screening tools that have the ability to accurately identify potentially malignant diseases in the incipient stages.

There are certain conditions that can be potentially malignant among different mucosal diseases. ${ }^{10}$ Identifying such lesions at the earliest help in attaining impressive results. ${ }^{11}$ These dysplastic areas are not usually visible to an unaided eye. This hinders the early stage detection of such lesions. Hence adjunctive techniques like the chemi luminescence and tissue auto fluorescence have been suggested for an accurate diagnosis. ${ }^{12}$ These light based investigations are advised by only 1 percent of the respondents of the present study. Scarce evidence to support the effectiveness of these adjunctive techniques ${ }^{12}$ may be the cause for very less usage of these techniques by the respondents of the study.

Diagnostic imaging for TMJ disorders is indicated for early assessment of symptoms and the differential diagnosis between TMD and inflammatory oral and maxillofacial conditions. ${ }^{3}$ These are advised by $20 \%$ of the dental practitioners. Low specificity of the conventional imaging modalities and high cost of the advanced ones may be the hindering factor. About 3\% of the practitioners in the present study believed that appliance therapy for patients with TMJ disorders is essential. Comprehensive examination to the TMJ and its related structures are required to choose proper appliance with fewer complications. ${ }^{14}$ Time constraint in examination may be obstructing the use of appliance and resulting in referral of those patients.

Pharmacological management with analgesics and anesthetics is considered to be the most efficient method by majority of the practitioners in the present study. These methods mainly aimed towards achieving symptomatic relief for the patients. The results of such treatments can be transient as the cause remains untreated.

Since some mucosal lesion may gradually develop into a malignancy in the lifetime of a patient [15], referral to an appropriate specialist is important. Thirty four percent of the respondents of the present study referred their patients to specialist routinely. In a study about the practices of general dentists concerning oral cancer, $98 \%$ dentists agreed that the patients should be referred to specialists in case of suspected malignancy. ${ }^{16}$ The misdiagnosis of various conditions can lead to improper referral; like TMJ disorders may be misdiagnosed as ear ache, which leads to referral to an ENT specialist.

In the present study, the clinicians referred $41 \%$ white and red lesions, $35 \%$ benign tumors, $12 \%$ oral cancers and $20 \%$ TMJ disorders to oral physicians. This reflects a lack of referring frequency of patients with oral mucosal lesions and TMJ disorders to oral physicians. Thus there is an evolving need to create awareness among general practitioners to refer those patients to oral medicine and radiology specialists.

The present study shows that there are conflicts between the awareness of different dental practitioners and their pattern of addressing different oral mucosal diseases and TMJ disorders. This accentuates the need of additional educational programs for the dental practitioners to keep them in harmony with the current and advanced diagnostic and therapeutic measures in the field of dentistry. It is perceptible that further study is enviable to recognize the barriers dentist experience to implement their knowledge in addressing different 
oral mucosal diseases and TMJ disorders. It would be favourable to conduct educational programs focusing on risk factor screening, habit counselling, and physical examination of the oral cavity and a review of the criteria for referral to a specialist for specific investigations, definitive diagnosis and treatment, especially to oral physicians.

\section{CONCLUSION}

There should be emphasis on workshops for general dental practitioners regarding the available chair side investigations for screening potentially malignant disorders and malignancy. The study also implies that the knowledge about diagnosis and referral of TMJ disorders need to be reinforced. The enormous scope of the specialty dental practice, especially oral medicine and radiology, should be underscored. Private practitioners should be invited for various CDEs, conventions and conferences for better understanding of the subject and also regarding the latest protocols for referral of the patients to specialists. It would be favourable to conduct educational programs focusing on risk factor screening, habit counselling, physical examination of the oral cavity and a review of the criteria for referral to a specialist for specific investigations, definitive diagnosis and treatment, especially to oral physicians.

\section{REFERENCES}

1. Stoopler ET, Sollecito TP. Oral Mucosal Diseases Evaluation and Management. Med Clin N Am 2014; 98: $1323-52$.

2. Dworkin SF, LeResche L, DeRouen T, Von Korff M. Assessing clinical signs of temporomandibular disorders: reliability of clinical examiners. J Prosthet Dent. 1990; 63: 574-9.

3. Nomura K, Vitti M, Oliveira AS, Chaves TC, Semprini M, Siessere S, et al. Use of the Fonseca's questionnaire to assess the prevalence and severity of temporomandibular disorders in Brazilian dental undergraduates. Braz Dent J 2007; 18:16-37.
4. Bhatnagar P, Rai S, Prabhat M. Prevalence study of oral mucosal lesions, mucosal variants, and treatment required for patients reporting to a dental school in North India: In accordance withWHO guidelines. J Family Community Med 2013; 20: 41-8.

5. Paolo CD, Costanzo GD, Iannetti G. Epidemiological analysis on 2375 patients with TMJ disorders: basic statistical aspects. Ann Stomatol 2013; 4: 161-9.

6. McLeod NM, Saeed NR, Ali EA. Oral cancer: delays in referral and diagnosis persist. Br Dent J 1998; 11: 681-4.

7. Awan KH. Oral Cancer: Early Detection is Crucial. J Int Oral Health 2014; 6: 1-2.

8. Stern I, Greenberg MS. Clinical Assessment of Patients with Orofacial Pain and Tempo romandibular Disorders. Dent Clin N Am 2013; 57: 393-404.

9. Lingen MW, Kalmar JR, Karrison T, Speight PM. Critical evaluation of diagnostic aids for the detection of oral cancer. Oral Oncol 2008; 44: 10-22.

10. Yardimci G, Kutlubay Z, Engin B, Tuzun Y. Precancerous lesions of oral mucosa. World J Clin Cases 2014; 2: 866-72.

11. Awan KH, Patil S, Islam SA, Jafer M. Early Detection of Oral Cancer Guidelines for Dental Practitioners. J Int Oral Health 2016; 8: 399-403.

12. Fedele S. Diagnostic aids in the screening of oral cancer. Head and Neck Oncol 2009; 1: 1-5.

13. Ferreira LA, Grossmann E, Januzzi E, Paula MVQ, Carvalho ACP. Diagnosis of temporomandibular joint disorders: indication of imaging exams .Braz J Otorhinolaryngol 2016; 82: 341-52.

14. Alqutaibi AY, Aboalrejal AN.Types of Occlusal Splint in Management of Temporomandibular Disorders (TMD). J Arthritis 2015; 4:176.

15. Speight PM, Farthing PM and Bouquot JE. The pathology of oral cancer and precancer. Current Diagnostic Pathology 1996; 3: 165-76.

16. Vijay KV, Suresan V. Knowledge, attitude and screening practices of general dentists concerning oral cancer in Bangalore city. Ind J of Cancer 2012; 49: $33-8$. 
Source of support: Nil, Conflict of interest: None declared

Cite this article as:

Anekar J, John IE, Raj AC, Nappalli D, Kumar KS, Baipadavu J. Dental Practitioners' Opinions and Methods in the Management of Oral Mucosal Lesions and TMJ Disorders in Dakshina

Kannada District: A Questionnaire Based Survey. Int Healthc Res J. 2018;2(7):168-175. doi: 10.26440/ihrj.v2i7.145

\section{AUTHOR AFFILIATIONS:}

1. Professor and Head, KVG Dental College and Hospital

2. Former Postgraduate Student, KVG Dental College

3. Professor and Head, MAHE Institute of Dental Sciences \& Hospital

4. Reader, KVG Dental College and Hospital

5. Assistant Professor, Amrita School of Dentistry

6. Senior Lecturer, KVG Dental College and Hospital

*Corresponding Author:

Dr. Ivin Elsa John

KVG Dental College

Sulia DK

\section{Karnataka 574327}

For article enquiry/author contact details, e-mail at: manuscriptenquiry.ihrj@gmail.com

\section{LEGENDS}

1.Duration of dental practice.

More than 1 years

$1-5$ years

5- 10 years

More than 10 years

2.Most common and rare oral lesions and disorders witnessed every month among oral ulcers, glossitis, white and red lesions, benign tumors, oral cancer and TMJ disorders.

3. Frequency of the different lesions witnessed every month.

Less than 5 / month

More than 5 / month

4. Investigations advised.

Blood investigations

Light based investigations

Exfoliative cytology

Biopsy

Radiographs

5. Treatment provided among pharmacological, surgical or other specific treatments.

6. If pharmacological treatment, drugs

7. Frequency of referral to specialists.

Not at all

Routinely

Selectively

8. If referred, preference of speciality among oral medicine and radiology and other specilaities

Figure 1. Questionnaire. 


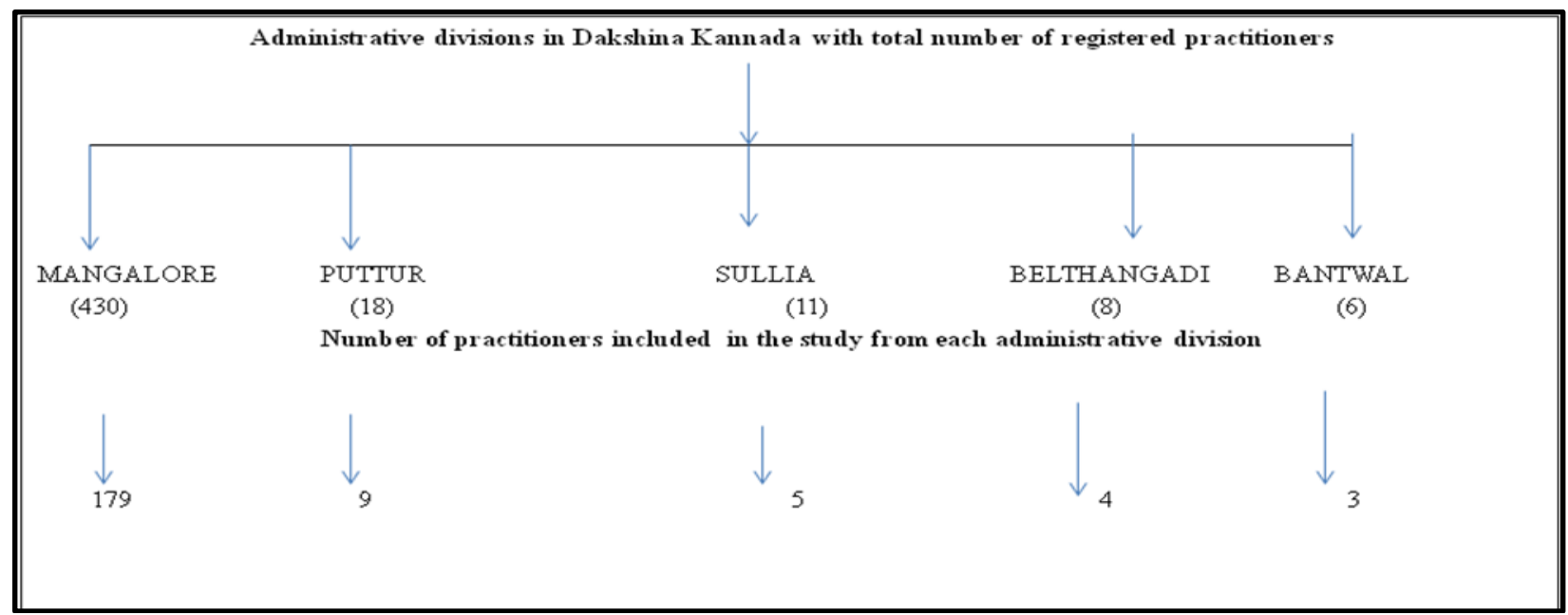

Figure 1(a). Sampling Frame.

\begin{tabular}{|c|c|c|}
\hline $\begin{array}{c}\text { MUCOSAL } \\
\text { LESIONS\& TMJ } \\
\text { DISORDERS }\end{array}$ & $\begin{array}{c}\text { MOST COMMON } \\
(\%)\end{array}$ & $\begin{array}{c}\text { RARE } \\
(\%)\end{array}$ \\
\hline Oral ulcers & 72 & 24 \\
\hline Glossitis & - & \\
\hline White and & 62 & 14 \\
\hline Red lesions & - & 26 \\
\hline Benign tumors & - & 24 \\
\hline Oral cancer & - & \\
\hline TMJ disorders & & \\
\hline
\end{tabular}

Table 1. Most common and rare oral mucosal lesions and TMJ disorders

\begin{tabular}{|c|c|c|}
\hline $\begin{array}{c}\text { MUCOSAL LESIONS AND } \\
\text { TMJ DISORDERS }\end{array}$ & $1-5$ & $>5$ \\
\hline Oral ulcers & 68 & 14 \\
\hline Glossitis & 11 & 1 \\
\hline White and red lesions & 56 & 12 \\
\hline Benign tumors & 5 & 1 \\
\hline
\end{tabular}

Table 2. Frequencies of different oral mucosal lesions and TMJ disorders per month 


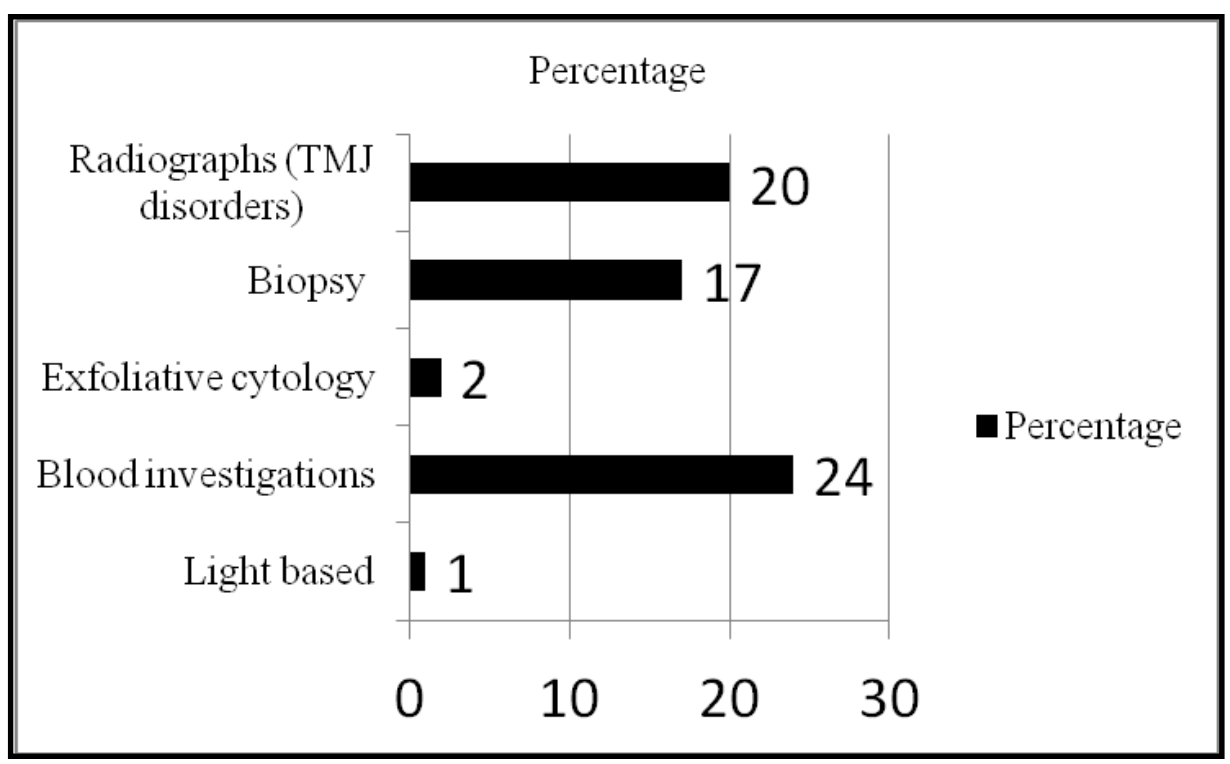

Figure 2. Investigations advised for oral mucosal lesions and TMJ disorders

\begin{tabular}{|c|l|}
\hline Oral ulcer & Local anesthetic and analgesics(Topical) \\
\hline Glossitis & Analgesics (Topical) \\
\hline White and red lesions & Anti fungals (Topical) ,multi vitamin (Tablets)
\end{tabular}

Table 3. Drugs prescribed for different oral mucosal lesions

\begin{tabular}{|c|c|}
\hline TYPE OF TREATMENT & PERCENTAGE \\
\hline Muscle relaxants & 83 \\
\hline Analgesics & 14 \\
\hline Appliance therapy & 3 \\
\hline
\end{tabular}

Table 4. Types of treatment for TMJ disorders 


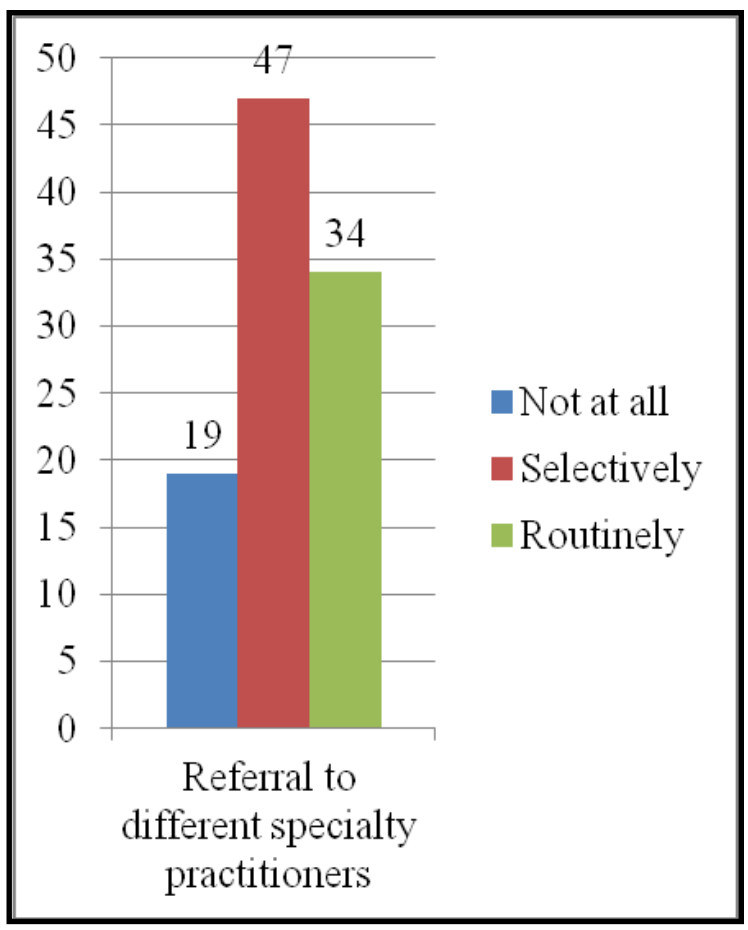

Figure 3. Referral frequencies to different specialty practitioners

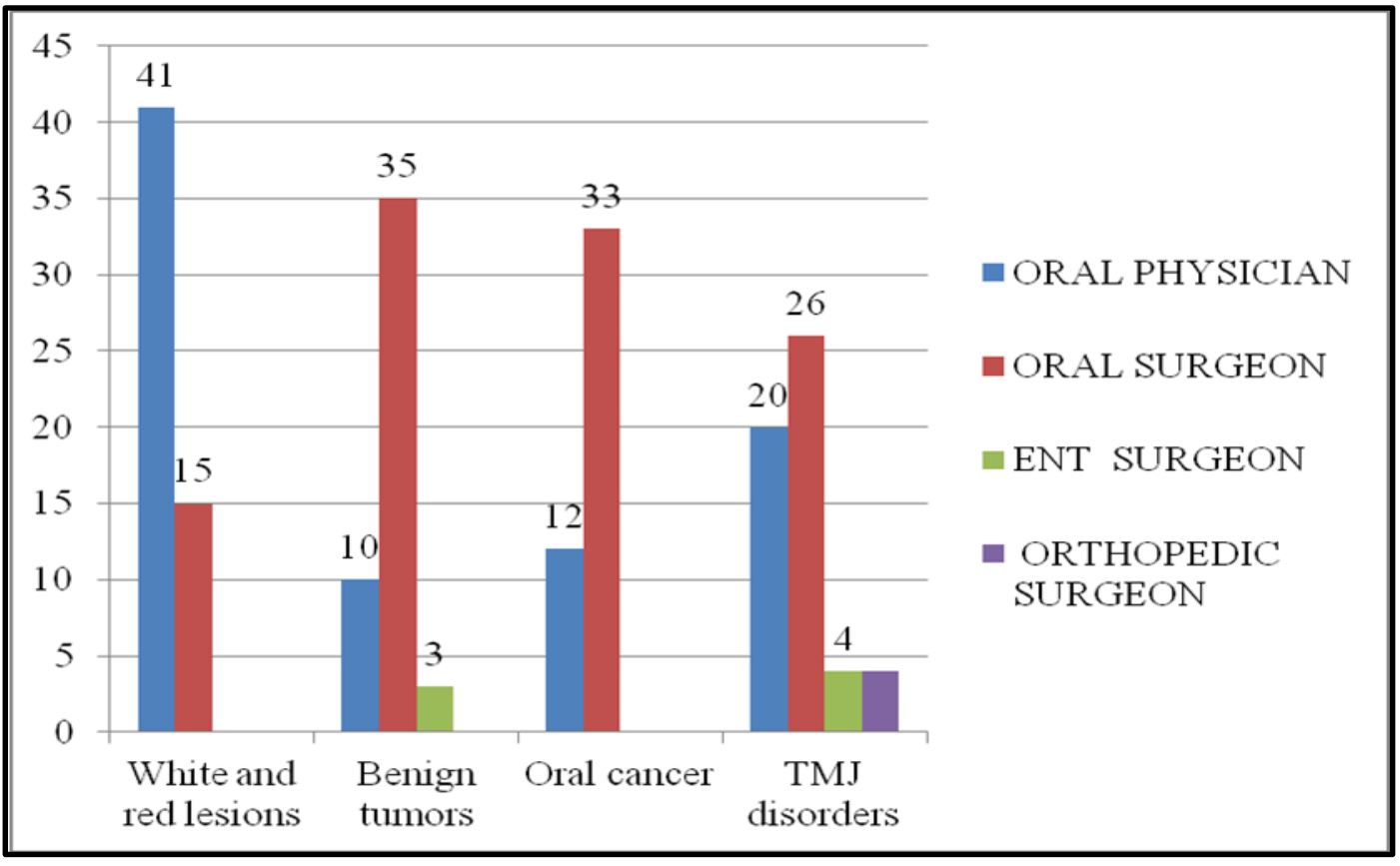

Figure 4. Pattern of referring to different specialty practitioners 